\section{Evaluación del crecimiento: estado nutricional}

\section{Growth evaluation: nutritional status}

Nelly F. Altamirano-Bustamante ${ }^{1}$ Myriam M. Altamirano-Bustamante ${ }^{2}$ Alejandro Valderrama-Hernánde $z^{1}$ Hortencia Montesinos-Correa ${ }^{1}$

${ }^{1}$ Servicio de Endocrinología, Instituto Nacional de Pediatría.

${ }^{2}$ Unidad de Investigación en Enfermedades Metabólicas, Centro Médico Nacional Siglo XXI, IMSS.

\section{INTRODUCCIÓN}

La evaluación adecuada del estado nutricional es un requisito indispensable en la vigilancia pediátrica del niño sano e imperativa en el niño enfermo, permite la identificación temprana de alteraciones y facilita la posibilidad de tratamiento oportuno; además de servir para la prevención primaria de desarrollo futuro de obesidad o de desnutrición y, por ende, de enfermedades crónicas degenerativas.

El peso es un marcador indirecto de la masa proteínica y de los almacenes de energía pero... ¿ ¿cuál es el peso corporal ideal? Establecer el peso ideal no es fácil teniendo en cuenta todos los factores involucrados. Además, ¿ideal en términos de qué?, ¿de salud, de estética, de rendimiento? El peso deseable debería ser aquel que dé lugar a una salud óptima y a un mínimo riesgo de enfermedades.

El pediatra debe mantener el peso deseable en cada etapa del paciente, pero fundamentalmente en los períodos críticos de crecimiento, para con ello realizar prevención primaria e identificar tempranamente a los niños en riesgo de desarrollar obesidad, diabetes mellitus tipo 2 o síndrome metabólico. El pediatra debe afrontar el reto de propiciar la expresión del máximo potencial de masa ósea y de masa magra, minimizar la expresión de genes de riesgo y permear así en cada niño su perfección como ser humano.

La evaluación del estado nutricional está enfocada a contestar las siguientes preguntas:
A. ¿Cuál es el estado nutricional del paciente?
B. ¿Cómo evaluarlo?

Recibido: 25 de septiembre, 2014

Aceptado: 10 de octubre 2014

Correspondencia: Nelly F. Altamirano Bustamante Instituto Nacional de Pediatría

Av. Insurgentes 3700-C CP0. 4530, México, D.F.

Tel.: 10840900 ext. 1823

nellyab34@gmail.com

Este artículo debe citarse como Altamirano-Bustamante NF, Altamirano-Bustamante MM, Valderrama-Hernández A, Montesinos-Correa $H$. Evaluación del crecimiento: estado nutricional. Acta Pediat Mex 2014;35:499-512. 
C. ¿El estado nutricional del paciente está alterado?

- $\quad$ ¿Tiene bajo peso?

- ¿Tiene sobrepeso?

- ¿Tiene obesidad?

- ¿Cómo es su distribución de grasa?

- ¿QQué tipo de obesidad tiene?

- ¿Cómo es la composición corporal?

- iTiene mayor cantidad de grasa?

D. ¿Desde cuándo se inició la alteración de su estado nutricional?

E. ¿Cuáles son las consecuencias actuales de la alteración del estado nutricional?

F. ¿Cuáles son las alternativas terapéuticas?

G. ¿Cuál es el pronóstico de las alteraciones del estado nutricional?

H. ¿Qué otras repercusiones futuras existen?

\section{A. ¿Cuál es el estado nutricional del paciente?}

La evaluación del estado nutricional y proveer una nutrición adecuada son componentes críticos en el seguimiento de niños sanos o enfermos.

\section{Parámetros poblacionales}

En el análisis del estado nutricional las dos variables más importantes son el peso en kilogramos y la talla en centímetros (o en metros y centímetros), los pliegues cutáneos y las medidas derivadas como el índice de masa corporal (peso $[\mathrm{kg}]) /$ talla $[\mathrm{m}]^{2}$ ); éstas se deben comparar con tablas y gráficas de crecimiento estándar que existen a escalas nacional e internacional. ${ }^{1}$

El peso expresado se grafica en parámetros de referencia, lo ideal es que cada población elabore las propias gráficas de acuerdo con sus características étnicas y geográficas. ${ }^{2,3}$ En niños de 0-24 meses debemos de utilizar las de la Organización Mundial de la Salud (WHO
Multicentre Growth Reference Study) y, a partir de los dos años de edad, las de los Centers for Disease Control and Prevention Atlanta 2000; cabe destacar que siempre debemos graficar longitudinalmente. ${ }^{4}$

En el análisis del crecimiento la edad cronológica exacta del paciente es un dato crucial. En prematuros es fundamental calcular la edad posnatal real que es imprescindible hasta los 24 meses para el peso y hasta los 3.5 años para la estatura. ${ }^{5}$

\section{Caso clínico}

Niño de dos años seis meses de edad que acude para evaluación del crecimiento: talla de pie de $80.7 \mathrm{~cm}$ y peso de $12.5 \mathrm{~kg}$.

\section{B. ¿Cómo evaluarlo?}

a) Centil poblacional (Figura 1).

Talla. Al ubicar la estatura expresada para edad y sexo se encuentra por debajo del centil 5 poblacional.

Peso: Al ubicarlo para edad y sexo está entre los centiles 10 y 25.

Índice de masa corporal (IMC): muestra la masa en función de la estatura y permite analizar las relaciones entre estas dos variables. El índice de masa corporal no refleja directamente exceso de grasa. Debe considerarse de manera individual y en el contexto de la actividad, ya que huesos densos y músculos bien desarrollados podrían dar un índice de masa corporal elevado. El paciente de nuestro caso tiene un índice de masa corporal por arriba del centil 95 para su edad y su sexo.

IMC $=$ peso $(\mathrm{kg}) /$ talla $(\mathrm{m})^{2}=12.5 /(0.807)^{2}=$ $19.2 \mathrm{~kg} / \mathrm{m}^{2}$ 
Diámetro de cintura: $55 \mathrm{~cm}$ ( > centil 75 para edad y sexo). Se mide a la mitad de la distancia entre el borde inferior de la última costilla y el borde superior de la cresta ilíaca. La distribución de la grasa permite distinguir dos somatotipos: el androide o en forma de manzana (distribución central) y el ginecoide en forma de pera (distribución periférica). La obesidad central se asocia con riesgo alto para desarrollar enfermedades crónicas degenerativas (Cuadro 1, Figura 2). ${ }^{6}$

\section{b) Puntaje Z (PZ) poblacional}

Describe más específicamente el estatus de las variables antropométricas individuales con respecto al estándar de referencia poblacional, WHO Multicentre Growth Reference Study Group 0-2 años de edad (Figura 3). ${ }^{7}$

$\mathrm{PZ}=\frac{M A-M A C 50}{D E}$

Donde:

MA: medida antropométrica

MAC50: medida antropométrica en el centil 50 para edad y sexo

DE: desviación estándar para edad y sexo

Así:

PZ de talla $=-3.05$

$P Z$ de peso $=-1.0$

$\mathrm{PZ}$ de índice de masa corporal $=1.9$

\section{Historia alimentaria}

Es un componente indispensable en la evaluación del estado nutricional. Nos proporciona información de la cantidad, calidad y composición de la alimentación: ingesta calórica, ingesta de macro- y de micronutrimentos, así como de los patrones y conductas familiares: número de comidas, colaciones, preferencias, factores psicosociales que inciden en la selección e ingesta de alimentos, alergias e intolerancia a alimentos; estatus socioeconómico y cultural. En nuestro medio evaluamos mediante el registro de cuatro días de alimentación que incluya dos días de fin de semana y dos días entre semana.

La ingesta calórica del paciente ejemplo es de 1750 calorías al día con la siguiente distribución; hidratos de carbono $49.7 \%$, proteínas $18 \%$ y grasas $30 \%$; con alta ingesta de hidratos de carbono simples $31 \%$ y de grasas saturadas $15.6 \%$, con buen aporte proteínico, así como de fibra (proteína de alto valor biológico 14.3\% y $11.5 \%$, respectivamente) y relación calcio/ fósforo $<1$ (0.94).

\section{C. ¿El estado nutricional del paciente está alterado?}

\section{Análisis}

\section{De acuerdo con parámetros centilares:}

El peso (entre los centiles 10 y 25) se encuentra más de dos carriles por arriba del centil de la talla $(<5)$ y el índice de masa corporal (> centil 95 para edad y sexo) permite diagnosticar obesidad de acuerdo con todos los criterios internacionales aceptados para obesidad en niños (Figura 4).

De acuerdo con el puntaje zeta:

El PZ del índice de masa corporal (1.9) está 4.95 por arriba del PZ de talla (-3.05) ver Figura 2.

\section{Composición corporal}

La impedancia bioeléctrica es un método simple, práctico y ampliamente utilizado para evaluar la composición corporal; se estima a través de la resistencia y reactancia de la masa total, el porcentaje de grasa y el de masa libre de grasa. 




Figura 1. Parámetros auxológicos poblacionales en la primera evaluación. (continúa en la siguiente página) 


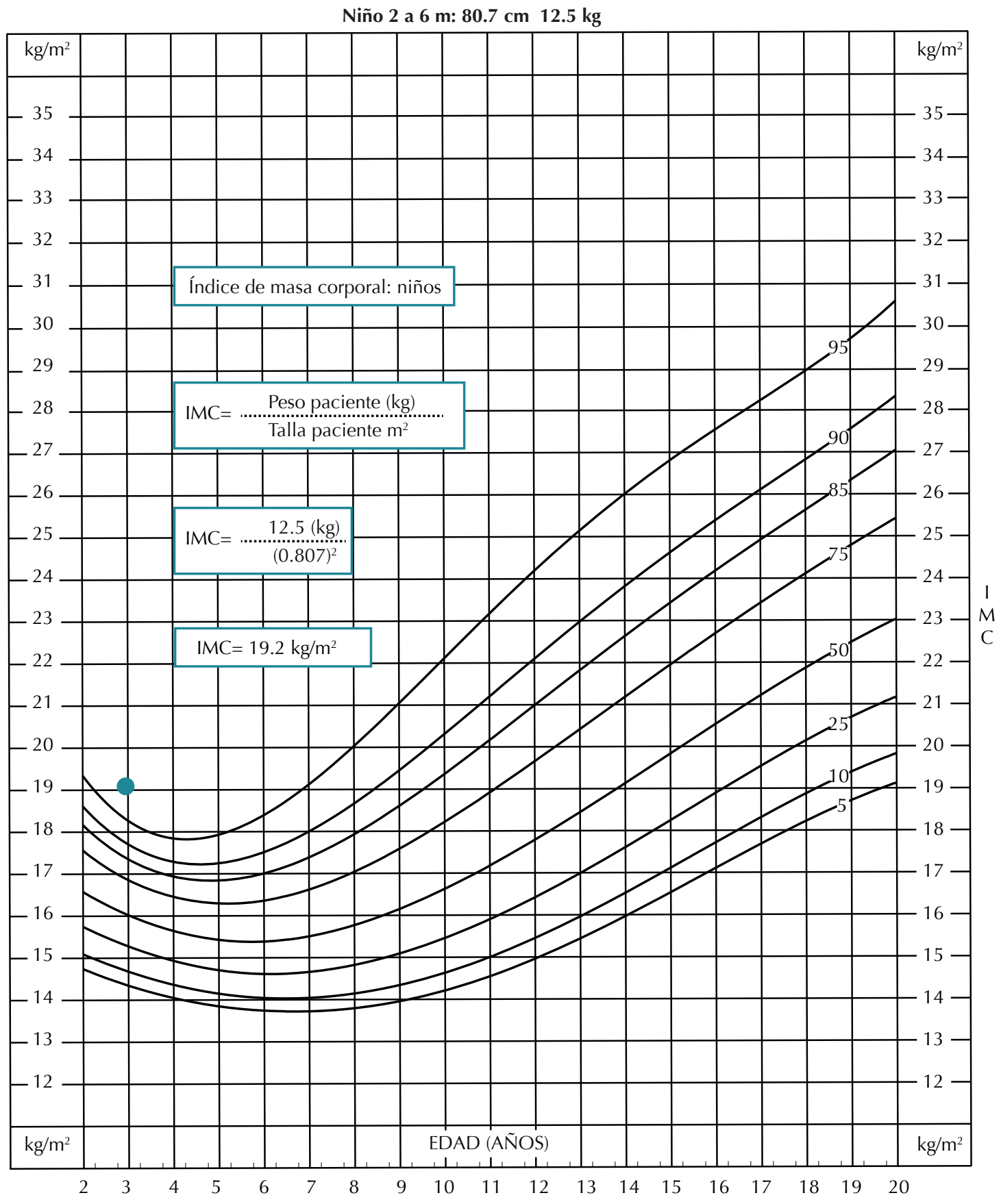

Figura 1. Parámetros auxológicos poblacionales en la primera evaluación. (continuación) 
Cuadro 1. Diámetro de cintura para niños y adolescentes mexicoamericanos

\begin{tabular}{cccccccccccc}
\hline & \multicolumn{9}{c}{ Centil para niños } & \multicolumn{9}{c}{ Centil para niñas } \\
& $\mathbf{1 0}$ & $\mathbf{2 5}$ & $\mathbf{5 0}$ & $\mathbf{7 5}$ & $\mathbf{9 0}$ & $\mathbf{1 0}$ & $\mathbf{2 5}$ & $\mathbf{5 0}$ & $\mathbf{7 5}$ & $\mathbf{9 0}$ \\
Intersección & $\mathbf{4 1 . 0}$ & $\mathbf{4 1 . 8}$ & $\mathbf{4 3 . 3}$ & $\mathbf{4 4 . 3}$ & $\mathbf{4 6 . 2}$ & $\mathbf{4 1 . 4}$ & $\mathbf{4 2 . 1}$ & $\mathbf{4 3 . 9}$ & $\mathbf{4 4 . 8}$ & $\mathbf{4 7 . 1}$ \\
Pendiente & $\mathbf{1 . 7}$ & $\mathbf{1 . 9}$ & $\mathbf{2 . 2}$ & $\mathbf{2 . 7}$ & $\mathbf{3 . 5}$ & $\mathbf{1 . 5}$ & $\mathbf{1 . 8}$ & $\mathbf{2 . 1}$ & $\mathbf{2 . 6}$ & $\mathbf{3 . 2}$ \\
Edad (años) & & & & & & & & & & \\
2 & 44.4 & 45.6 & 47.6 & $\mathbf{4 9 . 8}$ & 53.2 & 44.5 & 45.7 & 48.0 & 50.0 & 53.5 \\
3 & 46.1 & 47.5 & 49.8 & 52.5 & 56.7 & 46.0 & 47.4 & 50.1 & 52.6 & 56.7 \\
4 & 47.8 & 49.4 & 52.0 & 55.3 & 60.2 & 47.5 & 49.2 & 52.2 & 55.2 & 59.9 \\
5 & 49.5 & 51.3 & 54.2 & 58.0 & 63.6 & 49.0 & 51.0 & 54.2 & 57.8 & 63.0 \\
6 & 51.2 & 53.2 & 56.3 & 60.7 & 67.1 & 50.5 & 52.7 & 56.3 & 60.4 & 66.2 \\
7 & 52.9 & 55.1 & 58.5 & 63.4 & 70.6 & 52.0 & 54.5 & 58.4 & 63.0 & 69.4 \\
8 & 54.6 & 57.0 & 60.7 & 66.2 & 74.1 & 53.5 & 56.3 & 60.4 & 65.6 & 72.6 \\
9 & 56.3 & 58.9 & 62.9 & 68.9 & 77.6 & 55.0 & 58.0 & 62.5 & 68.2 & 75.8 \\
10 & 58.0 & 60.8 & 65.1 & 71.6 & 81.0 & 56.5 & 59.8 & 64.6 & 70.8 & 78.9 \\
11 & 59.7 & 62.7 & 67.2 & 74.4 & 84.5 & 58.1 & 61.6 & 66.6 & 73.4 & 82.1 \\
12 & 61.4 & 64.6 & 69.4 & 77.1 & 88.0 & 59.6 & 63.4 & 68.7 & 76.0 & 85.3 \\
13 & 63.1 & 66.5 & 71.6 & 79.8 & 91.5 & 61.1 & 65.1 & 70.8 & 78.6 & 88.5 \\
14 & 64.8 & 68.4 & 73.8 & 82.6 & 95.0 & 62.6 & 66.9 & 72.9 & 81.2 & 91.7 \\
15 & 66.5 & 70.3 & 76.0 & 85.3 & 98.4 & 64.1 & 68.7 & 74.9 & 83.8 & 94.8 \\
16 & 68.2 & 72.2 & 78.1 & 88.0 & 101.9 & 65.6 & 70.4 & 77.0 & 86.4 & 98.0 \\
17 & 69.9 & 74.1 & 80.3 & 90.7 & 105.4 & 67.1 & 72.2 & 79.1 & 89.0 & 101.2 \\
18 & 71.6 & 76.0 & 82.5 & 93.5 & 108.9 & 68.6 & 74.0 & 81.1 & 91.6 & 104.4
\end{tabular}

Modificado de Fernandez JR, Redden DT, Pietrobelli A, Allison DB. Waist circumference percentiles in nationally representative samples of African-American, European-American, and Mexican-American children and adolescents. J Pediatr $2004 ; 145: 439-444 .^{6}$

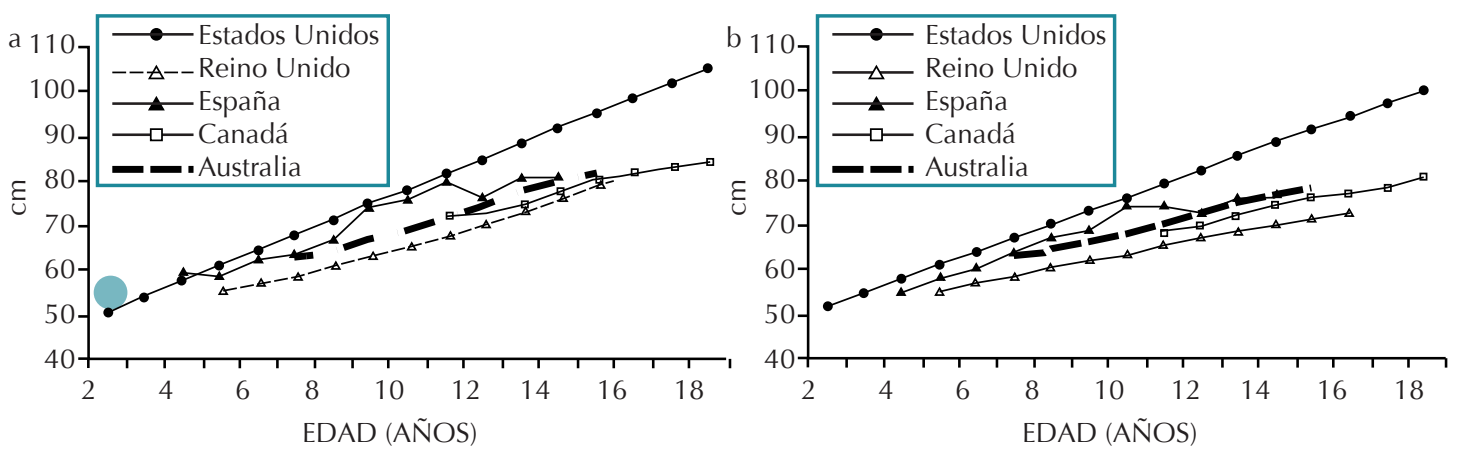

Figura 2. Percentil 50 de diámetro de cintura en diferentes poblaciones. Modificado de Brambilla P, Lissau I, Flodmark CE, Moreno LA, Widhalm K, Wabitsch M et.al. Metabolic risk-factor clustering estimation in children: to draw a line across pediatric metabolic syndrome. Int J Obesity 2007;31(4):591-600. ${ }^{8}$ 
Niño 2 a 6 m: $80.7 \mathrm{~cm} 12.5 \mathrm{~kg}$ IMC $19.2 \mathrm{~kg} / \mathrm{m}^{2}$

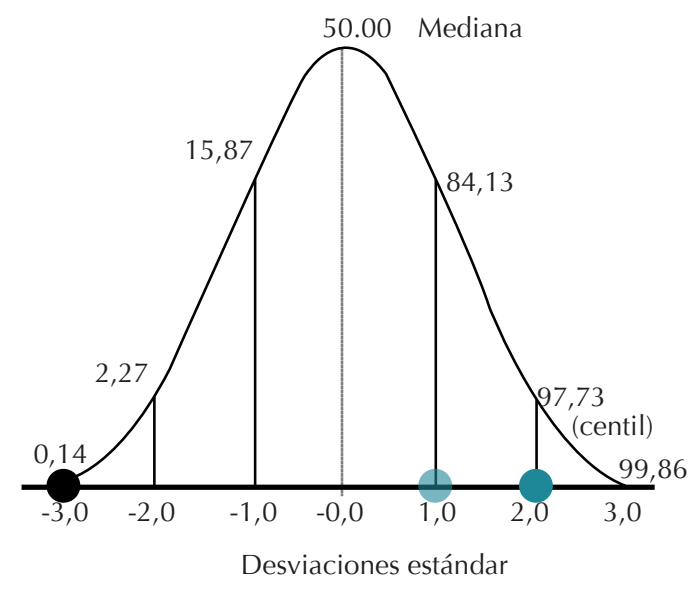

PZTalla $=-3.05 \mathrm{DE} P \mathrm{PZ}$ Peso $=-1.00 \mathrm{DE}$ $\mathrm{PZIMC}=1.9 \mathrm{DE}$

Figura 3. Parámetros de referencia poblacional en términos de puntuación zeta (desviación estándar) en la primera evaluación.

El porcentaje de grasa de nuestro ejemplo es de $24 \%$ y el de masa libre de grasa de $67 \%$.

El porcentaje máximo de grasa para la edad debiera ser $18 \%$ y el de masa libre de grasa $>70 \%$.

\section{Puntos clave}

De acuerdo con parámetros poblaciones el paciente tiene talla baja y obesidad central (Figuras 1-4):

- Talla expresada para edad y sexo menor del centil 5 poblacional.

- Talla expresada para edad y sexo por debajo de puntaje Z-2.

- Talla expresada para edad y sexo más de $4 \mathrm{~cm}$ por debajo del centil 50 familiar.

- Talla expresada para edad y sexo por debajo de puntaje Z-1 de la talla blanco familiar.
- Índice de masa corporal para edad y sexo por arriba del centil 95.

- Diámetro de cintura mayor que el centil 75 para edad y sexo para poblaciones reportadas. ${ }^{8}$

- Composición corporal alterada con mayor porcentaje de masa grasa y menor porcentaje de masa libre de grasa.

D. ¿Desde cuándo inició la alteración del estado nutricional?

Producto de la GI por cesárea a las 32 semanas por eclampsia y sufrimiento fetal agudo. ${ }^{9}$ Alimentación con seno materno más fórmula hasta los 6 meses. Los parámetros auxológicos se muestran en el Cuadro 2.

\section{Puntos clave}

La talla esperada para la edad gestacional era de $43 \mathrm{~cm}$ y el peso de $1780 \mathrm{~g}$ por lo que se integra el diagnóstico de prematuro con retraso de crecimiento intrauterino simétrico. ${ }^{10-14}$

La recuperación de la talla ha sido menor de la esperada, en tanto que la velocidad de ganancia en peso es mayor; es decir, el incremento en peso inició en el primer período crítico de crecimiento, donde no solamente hay hipertrofia sino también hiperplasia en los primeros dos años de vida. (Figuras 5 y 6 )

\section{E. ¿Cuáles son las consecuencias actuales de la alteración del estado nutricional?}

Por un lado la talla baja y la obesidad central evidencian la posibilidad de deficiencia de hormona de crecimiento $(\mathrm{GH})$ que se corrobora por velocidad de crecimiento baja, factor de crecimiento insulinoide tipo 1 (IGF I) $<-2$ DE y una prueba de hipoglucemia inducida por insulina con pico máximo de hormona de crecimiento de $6 \mathrm{ng} / \mathrm{mL}$. 
Niño 2 a 6 m: $80.7 \mathrm{~cm} 12.5 \mathrm{~kg}$ IMC $19.2 \mathrm{~kg} / \mathrm{m}^{2}$
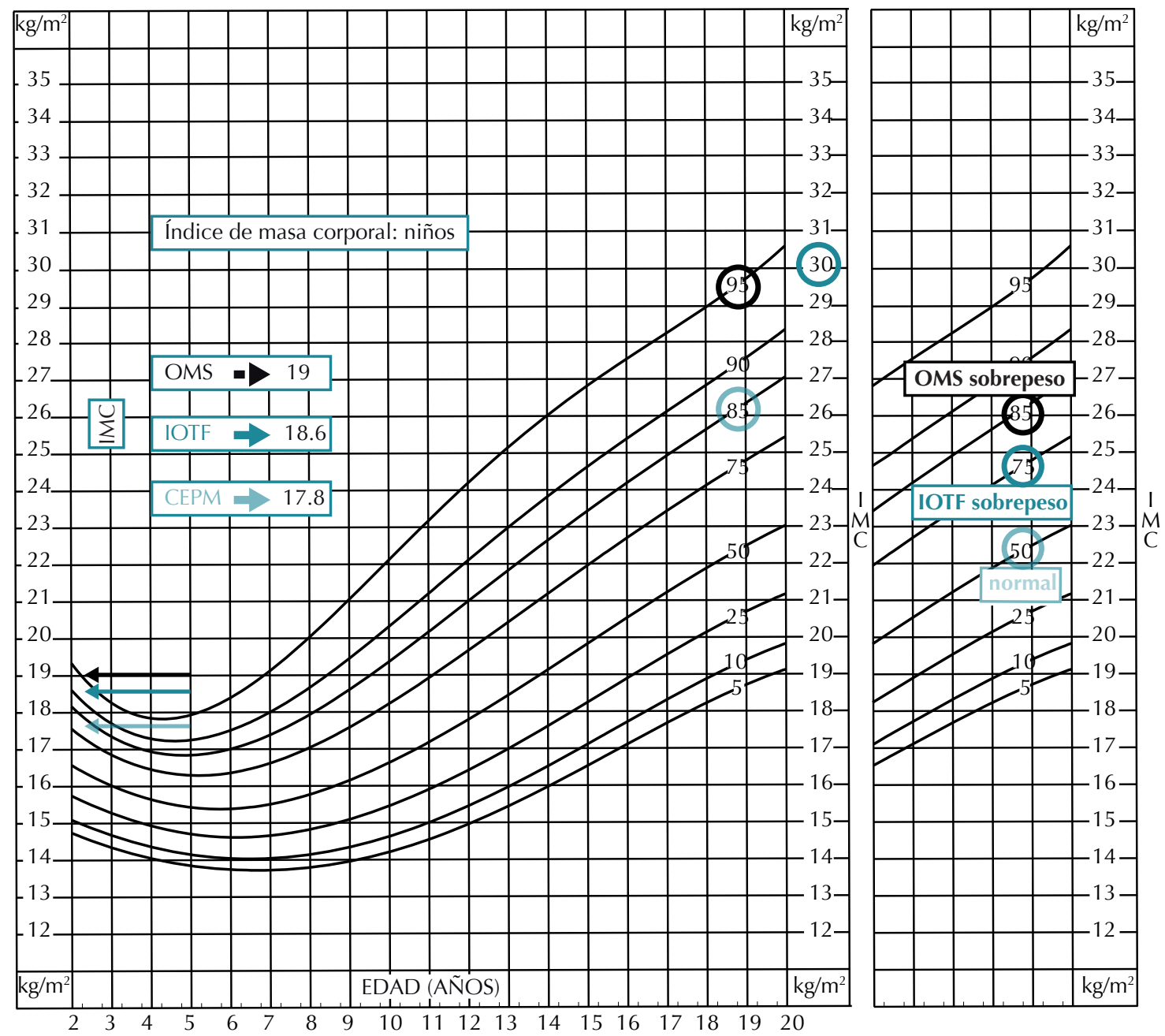

Figura 4. Diagnóstico de obesidad en niños para edad y sexo de acuerdo con parámetros mundiales aceptados: Organización Mundial de la Salud (OMS) (IMC $\geq$ centil 95); International Obesity Task Force (IOTF) (equivalente al IMC 30 del adulto) y Consenso de Endocrinólogos Pediatras Mexicanos (IMC $\geq$ centil 85). IMC: índice de masa corporal.

Esta deficiencia de hormona de crecimiento explica el biotipo del paciente: mayor porcentaje de grasa y menor de masa magra, razón por la que no se ha recuperado el retraso de crecimiento intrauterino en talla y continúa con velocidad de crecimiento baja.

\section{F. ¿Cuáles son las alternativas terapéuticas?}

Cambio terapéutico de estilo de vida de la familia: la madre mide $162 \mathrm{~cm}$, pesa $66 \mathrm{~kg}$, IMC $25.14 \mathrm{~kg} / \mathrm{m}^{2}$; el padre mide $170 \mathrm{~cm}$, pesa $80 \mathrm{~kg}$, IMC $27.68 \mathrm{~kg} / \mathrm{m}^{2}$. 
Cuadro 2. Seguimiento longitudinal de parámetros auxológicos

\begin{tabular}{|c|c|c|c|}
\hline $\begin{array}{l}\text { Edad (edad } \\
\text { corregida) }\end{array}$ & $\begin{array}{c}\text { Talla (cm)/ } \\
\text { PZ (DE) }\end{array}$ & $\begin{array}{c}\text { Peso (kg)/ } \\
\text { PZ (DE) }\end{array}$ & $\begin{array}{c}\mathrm{IMC} \\
\left(\mathrm{kg} / \mathrm{m}^{2}\right) / \mathrm{PZ} \\
(\mathrm{DE})\end{array}$ \\
\hline Recién nacido & $37 /-2.22$ & $1.150 /-1.89$ & - \\
\hline 1 año (10 m) & $69.7 /-1.57$ & 8.130/-1.09 & $\begin{array}{c}16.7 /-0.23 * \\
{[2.16} \\
\left.\mathrm{kg} / \mathrm{m}^{3}\right]\end{array}$ \\
\hline $\begin{array}{l}2 \text { años } 1 \\
(1 \text { a } 11 \mathrm{~m})\end{array}$ & 76.8/-3.39 & $11.2 /-0.57$ & \\
\hline $\begin{array}{c}2 \text { años } 6 \text { m } \\
(2 \text { a } 4 \text { m) }\end{array}$ & $* 80.7 /-3.01$ & $12.5 /-0.53$ & $19.2 / 2.38$ \\
\hline $\begin{array}{c}2 \text { años } 8 \mathrm{~m}^{* *} \\
(2 \text { a } 6 \mathrm{~m})\end{array}$ & $82.5 /-2.77^{*}$ & $12.6 /-0.56$ & $18.5 / 2.01$ \\
\hline 3 años 2 m (3 a) & $88.3 /-2.1 *$ & $12.3 /-1.46$ & $15.8 / 0.19$ \\
\hline \multicolumn{4}{|c|}{$\begin{array}{l}\text { DE: desviación estándar; IMC: índice de masa corporal; } \\
\text { PZ: puntaje Z. } \\
\text { *Puntaje Z de talla para edad corregida. } \\
\text { **Inició tratamiento con hormona de crecimiento biosin- } \\
\text { tética humana. }\end{array}$} \\
\hline
\end{tabular}

Tratamiento con hormona de crecimiento humana biosintética (HCHB) a razón de $0.33 \mathrm{mg} / \mathrm{kg} /$ semana. Inició a los 2 años 8 meses.

La respuesta en términos de velocidad de crecimiento muestra crecimiento de recuperación con mejoría del puntaje $Z$ de talla así como del puntaje $Z$ de peso y del índice de masa corporal.

\section{Puntos clave}

Los padres tienen sobrepeso, lo que favorece la presencia de obesidad por las preferencias por ciertos comestibles y conductas de búsqueda de alimentos.

\section{G. ¿Cuál es el pronóstico de las alteraciones del estado nutricional?}

Bueno, porque la hormona de crecimiento humana biosintética, además de mejorar la velocidad de crecimiento y recuperar talla, va a inducir cambios en la composición corporal con disminución de la masa grasa simultánea con ganancia de masa muscular; esto, aunado con el cambio terapéutico de estilo de vida va a provocar que los padres aprendan a proporcionar un plan de alimentación sano. Aporte calórico de acuerdo con edad, talla, con distribución de macronutrimentos: $50-55 \%$ de hidratos de carbono $(<10 \%$ de hidratos de carbono simples), $20 \%$ de proteínas y $30 \%$ de grasas $(<10 \%$ de grasas saturadas, $12 \%$ de poliinsaturadas y $10 \%$ de monoinsaturadas) con relación calcio/fósforo mayor de 1.

Muchos de los principales factores de riesgo asociados con productos pequeños para la edad gestacional son modificables. Las medidas preventivas antes y durante el embarazo recomendadas son: caminar diario en forma moderada, alimentación adecuada con ingesta de micronutrimentos, dormir ocho horas diarias, evitar exposición a químicos, evitar el consumo de tabaco y de alcohol pero, sobre todo, peso ideal para la talla preconcepcional y adecuada ganancia de peso durante la gestación.

\section{H. ¿Qué otras repercusiones futuras existen?}

Heredar a la siguiente generación los cambios epigenéticos que tienen riesgo de expresarse.

El factor crucial responsable del mayor riesgo de enfermedad metabólica, en etapas posteriores de la vida, es la velocidad de ganancia de peso en los primeros años de la vida posnatal. Existe mayor resistencia a la insulina ante el aumento del peso y de la adiposidad en los niños con retraso del crecimiento intrauterino.

Si nuestro paciente continúa con el tratamiento sustitutivo de hormona de crecimiento el pronóstico en talla final, pico máximo de masa ósea y composición corporal se espera exprese su máximo potencial. 


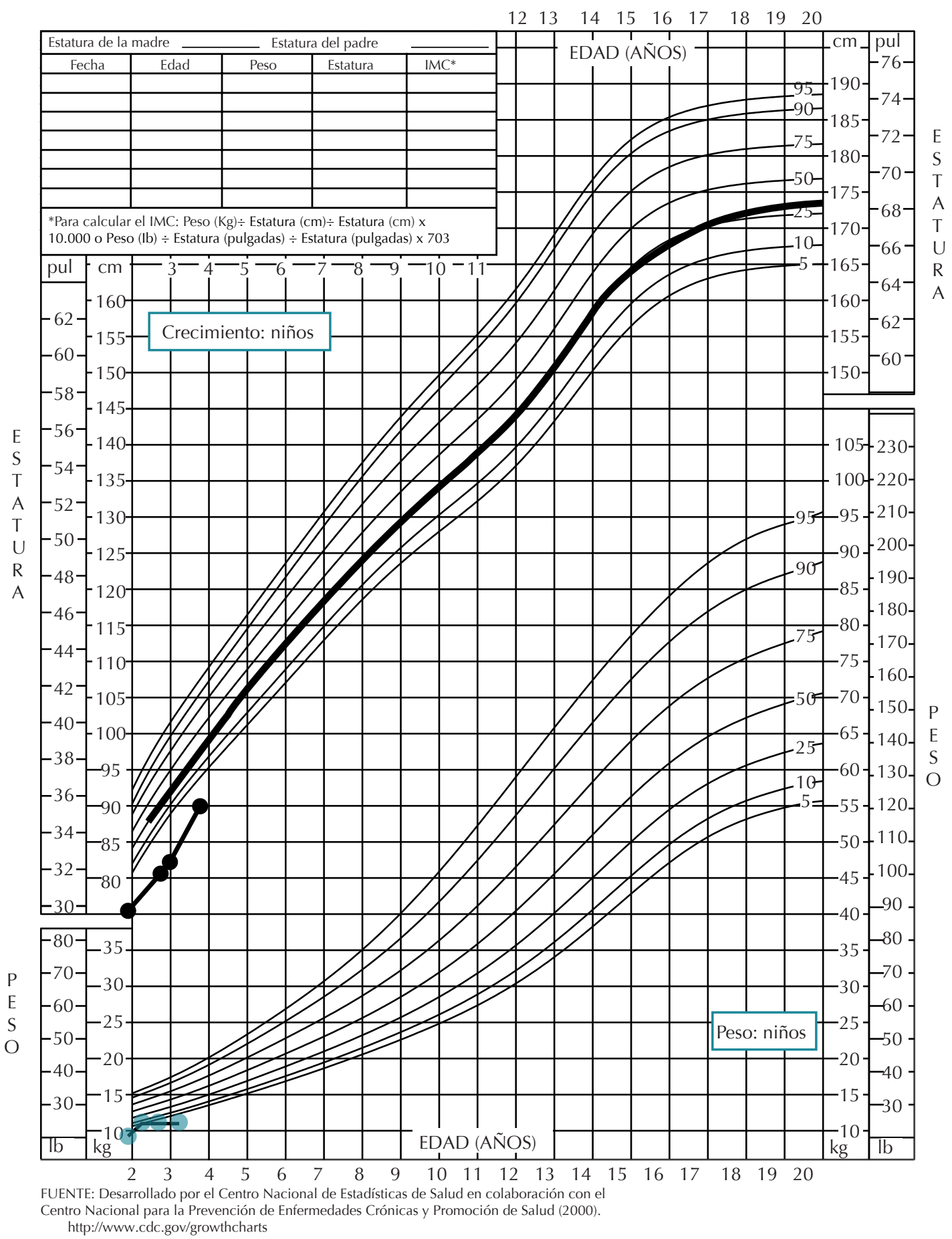

Figura 5. Seguimiento longitudinal de los parámetros auxológicos. (continúa en la siguiente página) 




Figura 5. Seguimiento longitudinal de los parámetros auxológicos. (continuación) 
Ya que existe una estrecha relación entre genes y ambiente es imperativo que el índice de masa corporal se mantenga por debajo del centil 25 para evitar el rebote temprano de la adiposidad y con ello disminuir el riesgo metabólico que por el antecedente de retraso de crecimiento intrauterino tiene programado.

El periodo de la concepción al nacimiento es un período de crecimiento rápido, de replicación celular, de diferenciación y de maduración funcional de órganos y sistemas. Estos procesos son muy sensibles a alteraciones en el medio intrauterino. El principal regulador del crecimiento fetal es la insulina. La programación fetal describe los mecanismos por los cuales un estímulo o un daño en ese período crítico del desarrollo tiene efectos tardíos o a lo largo de la vida. ${ }^{1}$ Un ambiente intrauterino adverso asociado con retraso del crecimiento fetal resulta en función y desarrollo alterado de la célula $\beta$, que puede finalizar en diabetes mellitus tipo 2 y otros factores de riesgo cardiovascular. Los mecanismos moleculares y celulares que alteran el desarrollo de la célula beta están relacionados con función mitocondrial anormal y alteraciones epigenéticas en genes clave de la célula $\beta$ (Figura 6). ${ }^{15,16}$

\section{CONCLUSIONES}

Los dos índices más utilizados en la actualidad para valorar el estado nutricional son el índice de masa corporal y la relación circunferencia de cintura/circunferencia de cadera. ${ }^{17,18}$

El incremento en parto prematuro indicado médicamente se acompaña de una sustancial disminución en la tasa de mortalidad perinatal, pero puede aumentar la posibilidad de evoluciones adversas posnatales con mayor morbilidad neonatal y consecuencias tardías tanto metabólicas como cognitivas en particular y afectar el neurodesarrollo en general.

La asociación de riesgo de diabetes mellitus tipo 2 en la vida adulta cuando hubo obesidad en la niñez asociada con otros factores de riesgo cardiovascular es: razón de posibilidades 11.5 (IC 95\%: 2.1-63) y de persistencia de síndrome metabólico en la vida adulta: razón de posibilidades 9.4 (IC 95\%: 4-22). Existen distintos criterios para el diagnóstico de síndrome metabólico que el pediatra debe conocer y vigilar (Cuadro 3). ${ }^{19}$

Cuadro 3. Criterios de diagnóstico para el síndrome metabólico. Se requiere la presencia de tres o más factores. No existe un criterio internacionalmente aceptado

\begin{tabular}{|c|c|c|c|c|}
\hline Componente & $\begin{array}{c}\text { Definición en adultos } \\
\text { ATP III }\end{array}$ & Centiles adultos & $\begin{array}{l}\text { Perfil pediátrico } \\
\text { Cook }\end{array}$ & $\begin{array}{c}\text { Perfil pediátrico ajustado } \\
\text { NHANES III }\end{array}$ \\
\hline $\begin{array}{l}\text { Obesidad central } \\
\text { (cintura) }\end{array}$ & $\begin{array}{l}102 \mathrm{~cm} \text { en varones } \\
88 \mathrm{~cm} \text { en mujeres }\end{array}$ & $\begin{array}{l}72 \text { en varones } \\
53 \text { en mujeres }\end{array}$ & $>$ centil 90 & $\begin{array}{c}\text { > centil } 75 \text { para edad, } \\
\text { sexo,raza }\end{array}$ \\
\hline $\begin{array}{l}\text { Glucosa } \\
\text { (ayunas) }\end{array}$ & $\geq 100 \mathrm{mg} / \mathrm{dL}$ & ND & $\geq 100 \mathrm{mg} / \mathrm{dL}$ & $\geq 100 \mathrm{mg} / \mathrm{dL}$ \\
\hline Hipertensión & $\geq 130 / 85 \mathrm{mmHg}$ & ND & $\begin{array}{l}\text { > centil } 90 \text { para } \\
\text { edad, sexo, raza }\end{array}$ & $\begin{array}{c}\text { > centil } 90 \text { para edad, sexo, } \\
\text { raza }\end{array}$ \\
\hline Triglicéridos & $>150 \mathrm{mg} / \mathrm{dL}$ & $\begin{array}{l}75 \text { en varones } \\
85 \text { en mujeres }\end{array}$ & $\geq 110 \mathrm{mg} / \mathrm{dL}$ & $\begin{array}{c}\text { > centil } 90 \text { para edad, sexo, } \\
\text { raza }\end{array}$ \\
\hline Colesterol HDL & $\begin{array}{l}<40 \mathrm{mg} / \mathrm{dL} \text { en varones } \\
<50 \mathrm{mg} / \mathrm{dL} \text { en mujeres }\end{array}$ & $<40$ & $<40 \mathrm{mg} / \mathrm{dL}$ & $<$ centil 10 para edad y raza \\
\hline
\end{tabular}

ATP III: Adult Treatment Panel of National Cholesterol Education Program.

NHANES III: National Health and Nutrition Examination Survey.

Modificado de: Shaibi GQ, Goran MI. Examining Metabolic Syndrome Definitions in Overweight Hispanic Youth: A Focus on Insulin Resistance. J Pediatr 2008;152:171-176. ${ }^{19}$ 
Altamirano-Bustamante NF et al. Evaluación del crecimiento


Figura 6. Momento de ganancia de peso. Períodos críticos de crecimiento. Obsérvese el centil 95 en ambas gráficas. En la etapa posnatal en el índice de masa corporal existe reaceleración a partir de los 4 años 6 meses, en tanto que en la velocidad de crecimiento se presenta sólo en la pubertad. Nótese que este desfase es más aparente por arriba del centil 25 del índice de masa corporal. 
Iniciar el embarazo en las mejores condiciones nutricionales y la adecuada ganancia de peso durante el embarazo, aunados con un cuidado prenatal oportuno y evitar hábitos nocivos como tabaquismo durante el embarazo, son las medidas preventivas que pueden disminuir la tasa de productos pequeños para la edad gestacional y de retrasos en el crecimiento intrauterino.

\section{REFERENCIAS}

1. CDC website: $z$ score data files, 2010 .

2. Marshall WA. Evaluation of growth rate in height over periods of less than one year. Arch Dis Child 1971;46:414-17.

3. Tanner JM, Whitehouse RH, Takaishi M. Standars from birth to maturity for height, weight, height velocity, and weight velocity for British children. Parts I y II, Arch Dis Child 1966;41(Part I-II):454, 613.

4. Mehta NM, Corkins MR, Lyman B, Malone A, Goday PS, Carney LN, Monczka JL, Plogsted SW, Schwenk WF: Defining pediatric malnutrition: a paradigm shift toward etiology-related definitions. JPEN J Parenter Enteral Nutr 2013;37:460-481.

5. Rojas Gabulli MI. Aspectos prácticos de la antropometría en pediatría. Paediátrica 2000;3:22-26.

6. Fernandez JR, Redden DT, Pietrobelli A, Allison DB: Waist circumference percentiles in nationally representative samples of African-American, European-American, and Mexican-American children and adolescents. J Pediatr 2004;145:439-444.

7. WHO Multicentre Growth Study website, 2006. http:// www.who.int/childgrowth/en

8. Brambilla P, Pietrobelli A. Abnormal body mass index trajectories in childhood and possible health consequences. Int J Obes (Lond) 2007;31:1761-1762.
9. Nacidos Demasiado Pronto. Grupo Redactor del Resumen Ejecutivo, Kinney, M.V., Howson, C.P, McDougall, L., \& Lawn, J.E. Resumen ejecutivo de Nacidos Demasiado Pronto: Informe de Acción Global sobre Nacimientos Prematuros. March of Dimes, PMNCH, Save the Children, Organización Mundial de la Salud, 2012.

10. Karlberg J, Albertsson-Wikland K. Growth in full-term small-for-gestational-age infants: from birth to final height. Pediatr Res 1995;38:733-739.

11. Hokken-Koelega AC, De Ridder MA, LemmenRJ, Den Hartog $H$, De Muinck Keizer-Schrama SM, Drop SL. Children born small for gestational age: do they catch-up? Pediatr Res 1995;38:267-271.

12. Wilcox AJ. On the importance and the unimportance of birth weight. Int J Epidemiol 2001;30:1233-1241.

13. Chard T, Yoong A, Macintosh M. The myth of fetal growth retardation at term. Br J Obstet Gynaecol 1993;100:1076-1081.

14. Wilcox MA, Johnson IR, Maynard PV, Smith SJ, Chilvers CED. The individualized birth weight ratio: A more logical outcome measure of pregnancy than birth weight alone. Br J Obstet Gynaecol 1993;100:342-347.

15. Jurado GE. El crecimiento intrauterino. Gac Med Mex 1971;102:255.

16. Jurado GE. La curva de crecimiento intrauterino como indicador de riesgo neonatal. I Simposio nacional sobre endocrinología e investigación pediátrica. Hosp Cent Milit.; 1985.

17. Nader PR, O'Brien M, Houts R, Bradley R, Belsky J, Crosnoe $R$, Friedman $S$, et al. Identifying risk for obesity in early childhood. Pediatrics 2006;118:e594-e601.

18. Norma Oficial Mexicana 007-SSA2-1993. Atención de la mujer en el embarazo, parto y puerperio y del recién nacido. Criterios y procedimientos para la prestación del servicio. Disponible en: Salud.gov.mx.

19. Shaibi G. \& Goran M. Examining Metabolic Syndrome Definitions in Overweight Hispanic Youth: A Focus on Insulin Resistance. The Journal of Pediatrics 2008;152(2):171-76. 